JOURNAL OF

SYMPLECTIC GEOMETRY

Volume 11, Number 3, 319-341, 2013

\title{
SYMPLECTIC MICROGEOMETRY III: MONOIDS
}

\author{
Alberto S. Cattaneo, Benoit Dherin and Alan Weinstein \\ We show that the category of Poisson manifolds and Poisson \\ maps, the category of symplectic microgroupoids and Lagrangian sub- \\ microgroupoids (as morphisms), and the category of monoids and \\ monoid morphisms in the microsymplectic category are equivalent sym- \\ metric monoidal categories.
}

\section{Contents}

1. Introduction $\quad 319$

2. Preliminaries $\quad 321$

2.1. Poisson geometry 321

2.2. Symplectic categories $\quad 322$

2.3. Categories of monoids $\quad 325$

2.4. Functorial quantization $\quad 325$

3. From Poisson manifolds to symplectic microgroupoids 327

3.1. Construction of $\Sigma \quad 327$

3.2. Micro-version 328

3.3. Changing the target category $\quad 330$

4. From symplectic microgroupoids to monoids 330

4.1. Construction of $M \quad 331$

4.2. Equivalence of categories 333

$\begin{array}{ll}\text { References } & 339\end{array}$

\section{Introduction}

This paper is a step toward a geometric and functorial approach to Poisson manifold quantization. At its core is a micro-version of the geometric approach to the quantization of Poisson manifolds by symplectic groupoids, 
as developed in $[\mathbf{7}, \mathbf{1 8}, \mathbf{1 9}, \mathbf{2 5}, \mathbf{2 9}, \mathbf{3 0}]$. This micro-version is essentially obtained by replacing groupoids by groupoid germs (or "microgroupoids") and canonical relations by symplectic micromorphisms, which are the special canonical relation germs introduced in $[\mathbf{8 , 9}]$. After these replacements, geometric constructions that are only functorial-looking in the world of groupoids and canonical relations become honest functors in the world of microgroupoids and symplectic micromorphisms. We will use quotation marks to remind us when we are not dealing with honest categories and functors.

In this paper, we are mostly concerned with developing a functorial micro-version of two constructions. The first construction is a symplectization "functor" $\Sigma$, as in Fernandes [11]. It replaces a Poisson manifold with its source 1-connected symplectic groupoid and a Poisson map with the Lagrangian subgroupoid integrating its graph, which is regarded as a coisotropic submanifold. The second functorial-looking construction, called $M$, replaces a symplectic groupoid with a monoid object in the symplectic "category" (i.e., the category of symplectic manifolds and canonical relations) and a Lagrangian subgroupoid in the product of two symplectic groupoids with a monoid map.

In the micro-world, $\Sigma$ and $M$ become honest functors, both of which are equivalences of symmetric monoidal categories. Moreover, they have additional advantages over their macro-world counterparts:

- The domain of $\Sigma$ is now the category of all Poisson manifolds and Poisson maps, as opposed to the macro-world where $\Sigma$ is only defined for the class of integrable Poisson manifolds;

- a monoid object in the microsymplectic category characterizes the symplectic microgroupoid it comes from completely, as opposed to the macro-world where the inverse map is not part of the monoid structure alone (see Example 6 and [4] for similar issues related to the inverse map).

The composition $Z=M \circ \Sigma$ (which we call the "Zakrzewski functor") establishes an equivalence of monoidal categories between the category of Poisson manifolds and Poisson maps and the category of monoids and monoid morphisms in the microsymplectic category. This is the main result of this paper. In itself, it gives a categorical formulation of Poisson geometry; it allows us to replace, in a functorial way, the Poisson bivector field with a commutative diagram in a monoidal category. Moreover, it gives an appropriate categorical framework for Poisson manifold functorial quantization by symplectic (micro)groupoid methods. This framework may also be relevant for functorial aspects of star-product dequantization as in [14-17]. 


\section{Preliminaries}

2.1. Poisson geometry. We fix here some notations and terminology.

A Poisson manifold is a smooth manifold $A$ whose algebra of smooth functions is endowed with a Poisson bracket; i.e., a Lie bracket $\{$,$\} on$ the space of smooth functions $C^{\infty}(A)$ that is a derivation in each argument. Thus, this bracket is completely determined by a bivector field $\Pi \in \Gamma\left(\wedge^{2} T A\right)$ defined by $\{f, g\}(x)=\langle d f \wedge d g, \Pi\rangle$, where $f, g \in C^{\infty}(A)$. A Poisson map $\phi: B \rightarrow A$ is a smooth map such that $\phi^{*}: C^{\infty}(A) \rightarrow C^{\infty}(B)$ respects the Poisson brackets.

We denote by Poisson the category of Poisson manifolds and Poisson maps. This category is monoidal (see $[\mathbf{2 2}]$ for a general reference on monoidal categories). Namely, the tensor product of two Poisson manifolds, denoted by $A \otimes B$, is defined by endowing the cartesian product $A \times B$ of the manifold with the Poisson bivector field $\Pi_{A} \oplus \Pi_{B}$ given by the sum of the Poisson bivector fields. On Poisson maps, the tensor product is simply the usual cartesian product of maps. The unit object of the category is the one-point manifold endowed with its unique (zero) Poisson structure. Poisson is in fact a symmetric monoidal category, whose symmetries $A \times B \rightarrow B \times A$ are the usual factor permutations $\epsilon_{A, B}(a, b)=(b, a)$.

A coisotropic submanifold $C$ of a Poisson manifold $(A, \Pi)$ is a submanifold satisfying the condition $\Pi^{\sharp}\left(N^{*}(C)\right) \subset T C$, where $\Pi^{\sharp}: T^{*} A \rightarrow T A$ is the vector bundle map $\Pi^{\sharp}(\nu)=\Pi(\nu,-)$ and $N^{*}(C) \subset T^{*} A$ is the conormal bundle of $C$. Coisotropic submanifolds play a role similar to that of Lagrangian submanifolds in symplectic geometry: Namely, a smooth map $\phi: B \rightarrow A$ is Poisson if and only if its graph, denoted by gr $\phi$, is a coisotropic submanifold of $A \otimes \bar{B}$, where $\bar{B}$ denotes the dual Poisson manifold of $(B, \Pi)$, which is the manifold $B$ endowed with the opposite Poisson structure $-\Pi$.

There is a notion of integration for Poisson manifolds by symplectic groupoids that generalizes the integration of Lie algebras by Lie groups. A symplectic groupoid is a Lie groupoid $G \rightrightarrows A$ (see [21] for a general reference on Lie groupoids) whose total space $G$ is a symplectic manifold and such that the graph of the groupoid product $m: G \times{ }_{A} G \longrightarrow G$ is a Lagrangian submanifold of $\bar{G} \times \bar{G} \times G$. A symplectic groupoid endows its unit space $A$ with a unique Poisson structure such that the target map $s: G \rightarrow A$ (resp. the source map $t: G \rightarrow A$ ) is Poisson (resp. anti-Poisson). We say that $G \rightrightarrows A$ integrates the Poisson manifold $(A, \Pi)$. A morphism of symplectic groupoids is a morphism of groupoids that is a symplectomorphism.

Let us write SymplGpd for the category of symplectic groupoids and symplectic groupoid morphisms (i.e., symplectomorphisms that also are groupoid morphisms). As in the category of Poisson manifolds, the cartesian product of manifolds endows the category of symplectic groupoids with a symmetric monoidal structure. One also defines the dual symplectic 
groupoid $\overline{G \rightrightarrows A}$ of a symplectic groupoid $(G, \omega) \rightrightarrows A$ as the the symplectic groupoid $(G,-\omega) \rightrightarrows A$ (which can also be obtained by interchanging the source and target while "reversing" the product law). If the symplectic groupoid integrates $A$, its dual integrates $\bar{A}$. Similarly, if the symplectic groupoid $G \rightrightarrows A$ integrates the Poisson manifold $\left(A, \Pi_{A}\right)$ and the symplectic groupoid $H \rightrightarrows B$ integrates the Poisson manifold $\left(B, \Pi_{B}\right)$, then the symplectic groupoid product $G \times H \rightrightarrows A \times B$ integrates the Poisson manifold product $\left(A \times B, \Pi_{A} \oplus \Pi_{B}\right)$.

Not all Poisson manifolds can be integrated by symplectic groupoids. The ones which can are called integrable. All the source 1-connected symplectic groupoids integrating an integrable Poisson manifold are isomorphic to the one resulting from the following construction in terms of homotopy classes of paths $[\mathbf{6 , 1 0}$. Let $(A, \Pi)$ be an integrable Poisson manifold. Consider the space $\mathcal{P}\left(T^{*} A\right)$ of cotangent paths; that is, the set of paths $g:[0,1] \rightarrow T^{*} A$ such that

$$
p(t)=\Pi^{\sharp}(x(t)) \dot{x}(t), \quad \text { where } g(t)=(p(t), x(t)),
$$

where $x:[0,1] \rightarrow A$ is the base map. The source 1 -connected symplectic groupoid of $(A, \Pi)$ can be realized as the quotient of the set of cotangent paths by a homotopy relation $\sim$ that fixes the end-points of the $x$-component of the cotangent path (see $[\mathbf{6 , 1 0}]$ for a definition of this relation). We denote by $\Sigma(A)$ this quotient and by $[g]$ the homotopy class of $g . \Sigma(A)$ is a symplectic groupoid over $A$ whose source and target maps $s, t: \Sigma(A) \rightrightarrows A$ are given by the endpoints of the path projection on the base: $s([g])=x(0)$ and $t([g])=x(1)$. The groupoid product is given by concatenation of paths $[g]\left[g^{\prime}\right]=\left[g g^{\prime}\right]$, where $g \in[g]$ and $g^{\prime} \in\left[g^{\prime}\right]$ are two representatives whose ends $t([g])=s\left(\left[g^{\prime}\right]\right)$ match smoothly and where

$$
\left(g g^{\prime}\right)(t)=\left\{\begin{array}{cc}
2 g(2 t), & 0 \leq t \leq \frac{1}{2} \\
2 g^{\prime}(2 t-1), & \frac{1}{2}<t \leq 1
\end{array}\right.
$$

From now on, we will reserve the notation $\Sigma(A) \rightrightarrows A$ for the source 1-connected symplectic groupoid integrating $(A, \Pi)$ coming from the construction above.

Note that $\Sigma(A)$ always exists but not necessarily as a manifold: for nonintegrable Poisson manifolds, $\Sigma(A)$ can be realized as a stack (see [24])

2.2. Symplectic categories. Let us fix here a coherent notation for the various categories we will be dealing with. First of all, we have the following:

- Sympl is the usual symplectic category of symplectic manifolds and symplectomorphisms,

- Sympl ${ }^{\text {ext }}$ is the extended symplectic "category," where symplectomorphisms are replaced by canonical relations.

While the former is an honest category, the latter is not: the composition of two canonical relations may fail to produce again a canonical relation. 
There are two honest categories obtained by considering symplectic microfolds instead of symplectic manifolds.

Let us recall a few basic definitions from $[\mathbf{8}, \mathbf{9}]$.

A local manifold pair $(M, A)$ consists of a manifold $M$ and a submanifold $A \subset M$, called the core. Two manifold pairs $(M, A)$ and $(N, B)$ are said to be equivalent if $A=B$ and if there is a third manifold pair $(U, A)$ such that $U$ is an open subset in both $M$ and $N$ simultaneously. Note that we require equality of neighborhoods and not merely diffeomorphism.

A microfold is an equivalence class of a local pairs $(M, A)$. We denote these equivalence classes either by $[M, A]$ or by $([M], A)$. Sometimes, $[M]$ will be referred to as a manifold germ around $A$.

A submicrofold of a microfold $[M, A]$ is a microfold $[N, B]$ such that $N \subset M$ and $B \subset A$.

A symplectic microfold $[M, A]$ is a germ of a symplectic manifold $M$ around a Lagrangian submanifold $A \subset M$ called the core of the symplectic microfold.

A lagrangian submicrofold $[L, X]$ of a symplectic microfold $[M, A]$ is a microfold such that there are representatives $L$ of $[L]$ and $M$ of $[M]$ such that $L$ is Lagrangian in $M$.

A canonical relation (germ) from a symplectic microfold $[M, A]$ to a symplectic microfold $[N, B]$ is a Lagrangian submicrofold $[V, X]$ of $[\bar{M} \times N, A \times B]$, where $\bar{M}$ denotes the symplectic manifold with opposite symplectic form.

Now we consider canonical relations $[V, X]$ from $[M, A]$ to $[N, B]$ whose cores $X$ have a special form: Namely, consider a smooth map $\phi: B \rightarrow A$. The graph of $\phi$, which we denote by $\operatorname{gr} \phi$, is an embedded submanifold of $A \times B$, which we regard as an embedded submanifold of $M \times N$, since $A \times B$ is naturally embedded in $M \times N$. This allows us to consider canonical relations from $[M, A]$ to $[N, B]$ of the form $[V, \operatorname{gr} \phi]$.

A symplectic micromorphism ${ }^{1}$ from $[M, A]$ to $[N, B]$ is a canonical relation $[V$, gr $\phi]$, where $\phi$ is a smooth map from $B$ to $A$ as above, that intersects the core $A \times B$ cleanly in gr $\phi$.

For symplectic micromorphisms, we will exclusively use the notation

$$
([V], \phi):[M, A] \rightarrow[N, B]
$$

since they are actual morphisms of a category, the microsymplectic category, as shown in $[8]$.

\footnotetext{
${ }^{1}$ This definition is not the original one given in $[8]$ but an equivalent one as stated in [9, Thm. 17].
} 
We denote by

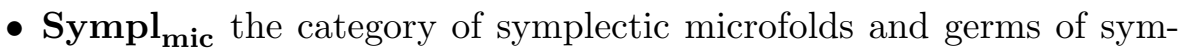
plectomorphisms,

- Sympl mic the category of the symplectic microfolds and symplectic micromorphisms.

We call this last category the microsymplectic category.

Each of the categories above is symmetric monoidal. The symmetric monoidal structure for $\mathbf{S y m p l}$ and $\mathbf{S y m p l} \mathbf{m i c}_{\text {mic }}$ comes from the usual cartesian product of sets and maps, while the symmetries

$$
\epsilon_{M, N}: M \times N \rightarrow N \times M .
$$

are given by the usual factor permutations $\epsilon_{M, N}(m, n)=(n, m)$.

As for Sympl ${ }^{\text {ext }}$, the symmetric monoidal structure comes from the one on the category of sets and binary relations: the tensor product on objects is still given by the cartesian product of the underlying sets, while the tensor product between a canonical relation $V$ from $M$ to $N$ and a canonical relation $W$ from $P$ to $Q$ is the canonical relation

$$
V \otimes W:=\left(\operatorname{id}_{M} \times \epsilon_{N, P} \times \operatorname{id}_{Q}\right)(V \times W)
$$

from $M \times P$ to $N \times Q$; i.e., the canonical relation $V \times W$ with the middle factors permuted. The symmetries are given by the graphs of the factor permutations

$$
\operatorname{gr} \epsilon_{M, N}=\{((m, n),(n, m)):(m, n) \in M \times N\},
$$

which are canonical relations from $M \times N$ to $N \times M$ for every symplectic manifold $M$ and $N$. The unit object $E$ is the one-point symplectic manifold, which we will also denote by $\{\star\}$.

We obtain the symmetric monoidal structure in the microsymplectic category by going to the representatives. The tensor product of two symplectic microfolds is given by the usual cartesian product of their underlying representatives:

$$
[M, A] \otimes[N, B]:=[M \times N, A \times B] .
$$

The tensor product of two symplectic micromorphisms is simply

$$
([V], \phi) \otimes([W], \psi):=([V \otimes W], \phi \times \psi),
$$

where $V \otimes W$ is the tensor product of the canonical relation representatives as above. Again, the symmetries are given by the graphs of the factor permutation maps. For the unit object $E$, we can take the symplectic microfold associated with the cotangent bundle of the one-point manifold $T^{*}\{\star\} \simeq\{0\} \times\{\star\}$. Observe that, in the microsymplectic category, there is only one morphism from the unit object to any given symplectic microfold:

$$
e_{M}:=\left([\{\star\} \times A], p r_{A}\right): E \rightarrow[M, A],
$$

where $p r_{A}$ is the unique map from $A$ to the one-point manifold. 
2.3. Categories of monoids. Each of the symplectic categories considered in the previous paragraph is symmetric monoidal, and thus possesses an associated category of monoids.

Recall that a monoid object (or monoid for short) in a monoidal category $(\mathcal{C}, \otimes, E)$ with neutral object $\mathbf{E}$ is a triple $(C, \mu, \mathbf{e})$ consisting of an object $C$, a morphism $\mu: C \otimes C \rightarrow C$, called the product, and a morphism $E: \mathbf{E} \rightarrow C$, called the unit. These morphisms satisfy the associativity and unitality axioms: i.e., respectively,

$$
\begin{gathered}
\mu \circ(\mu \otimes \mathrm{id})=\mu \circ(\mathrm{id} \otimes \mu) \\
\mu \circ(e \otimes \mathrm{id})=\mu \circ(\mathrm{id} \otimes e)=\mathrm{id} .
\end{gathered}
$$

A monoid morphism from a monoid object $\left(C, \mu_{c}, e_{c}\right)$ to a monoid object $\left(D, \mu_{D}, e_{D}\right)$ is a morphism $T: C \rightarrow D$ that respects the products and the units: i.e.,

$$
\begin{gathered}
T \circ \mu_{C}=\mu_{D} \circ(T \otimes T), \\
T \circ e_{C}=e_{D} \circ T .
\end{gathered}
$$

The monoid objects in $\mathcal{C}$ together with the monoid morphisms form a category $\operatorname{Mon}(\mathcal{C})$. In the sequel, we will be particularly interested in $\mathbf{M o n}\left(\mathbf{S y m p l}^{\mathbf{e x t}}\right)$, which is only a "category", and its micro-version $\operatorname{Mon}\left(\mathbf{S y m p l}_{\text {mic }}^{\text {ext }}\right)$, which is an honest category.

The category of monoids in a symmetric monoidal category $(\mathcal{C}, \otimes, \epsilon, E)$ is again monoidal symmetric. The tensor product on objects is given by

$$
\left(C, \mu_{C}, e_{C}\right) \otimes\left(D, \mu_{D}, e_{D}\right):=\left(C \otimes D, \mu_{C \otimes D}, e_{C} \otimes e_{D}\right),
$$

where

$$
\mu_{C \otimes D}:=\left(\mu_{C} \otimes \mu_{D}\right) \circ\left(\operatorname{id}_{C} \otimes \epsilon_{C, D} \otimes \operatorname{id}_{D}\right),
$$

while the tensor product on monoid morphisms is the tensor product of the underlying morphisms in $\mathcal{C}$. Since a monoidal category comes with an isomorphism $E \otimes E \rightarrow E$, the unit object in $\mathcal{C}$ is also the unit object in its category of monoids.

2.4. Functorial quantization. Symplectic groupoids were first introduced as a potential tool to be used along with geometric quantization in order to

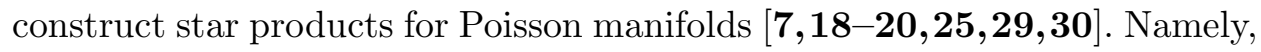


geometric quantization establishes a dictionary between symplectic geometry and linear algebra ${ }^{2}$

$$
\begin{array}{clll}
\text { Symplectic manifold } & M & \rightsquigarrow & \text { vector space } Q(M) \\
\text { Lagrangian submanifold } L \subset M & \rightsquigarrow & \text { vector } Q(L) \in Q(M) \\
\text { Opposite } \bar{M} & & \rightsquigarrow & \text { dual } Q(M)^{*} \\
\text { Cartesian product } M \times N & \rightsquigarrow & \text { tensor product } Q(M) \otimes Q(N)
\end{array}
$$

Implying the additional entry

$$
\text { Canonical relation } L \subset \bar{M} \times N \rightsquigarrow \text { linear map } \quad Q(L): Q(M) \rightarrow Q(N) .
$$

This suggests the possibility of a monoidal "functor"

$$
Q: \mathbf{S y m p l}^{\text {ext }} \rightarrow \operatorname{Vect}_{\mathbb{C}},
$$

(the quotation marks remind us that the source of this "functor" is only a "category"), which would induce a "functor" between the corresponding categories of monoids

$$
Q: \operatorname{Mon}\left(\mathbf{S y m p l}^{\mathrm{ext}}\right) \stackrel{Q}{\longrightarrow} \mathbf{A l g}_{\mathbb{C}}
$$

where $\mathbf{A l g}_{\mathbb{C}}$ is the category of monoids in $\operatorname{Vect}_{\mathbb{C}}$, or, in other words, the category of unital associative algebras and unital algebra maps over $\mathbb{C}$.

Now, the product graph gr $m \subset \bar{G} \times \bar{G} \times G$ of a symplectic groupoid $G \rightrightarrows A$ together with its unit space $A$ (regarded as a canonical relation from the onepoint symplectic manifold to $G$ ) produces a monoid object $(G, \operatorname{gr} m, A)$ in the symplectic "category." The image of this monoid object by $Q$ should yield a unital associative algebra $(Q(G), Q(\operatorname{gr} m), Q(A))$, which is to be thought of as the quantum algebra that quantizes the Poisson manifold $(A, \Pi)$ induced by the symplectic groupoid.

The strategy above has been carried out successfully in some concrete examples and special classes of integrable Poisson manifolds $[\mathbf{2}, \mathbf{3}, \mathbf{1 2}, \mathbf{1 3}$, 23, 25-27]. However, with this approach (as opposed to deformation quantization for instance), non integrable Poisson manifolds are discarded from the start, and functoriality issues, due to the ill-behaved composition of canonical relations, have to be faced. As we shall see in the coming sections, the use of the microsymplectic category improves the situation in these respects. Namely, any monoidal functor

$$
\text { Sympl }_{\text {mic }}^{\text {ext }} \stackrel{Q}{\longrightarrow} \text { Vect }_{\mathbb{C}}
$$

\footnotetext{
${ }^{2}$ Actually, $Q(L)$ is a set of vectors in $Q(M)$ unless $L$ is "enhanced" by a half density. For simplicity, we leave aside this aspect of the discussion, and we refer the reader to [1] for a full account.
} 
respecting duals and tensor products, would yield a general quantization scheme $^{3}$ for Poisson manifolds. The question of the functoriality of these quantization schemes is closely related to the possibility of enlarging the category of local symplectic groupoids into a category allowing us to regard the integration of Poisson manifolds by local symplectic groupoids as a functor. In fact, these ideas point to a local version of the early work of Zakrzewski $[\mathbf{2 9}, \mathbf{3 0}]$ and the more recent symplectization "functor" studied by Fernandes in $[\mathbf{1 1}]$ in the global case.

Let us conclude this paragraph by mentioning yet another way to circumvent the functorial issues raised by the ill-defined composition of canonical relations. Instead of the microsymplectic category, one could use the WW-category introduced by Wehrheim and Woodward in [28], which contains and enlarges the symplectic "category" so as to obtain an honest category. A symplectic groupoid can be then identified with a monoid object (endowed with some additional structures such as a $\star$-operation as in Zakrzewski $[\mathbf{2 9}, \mathbf{3 0}]$ ) in this category.

\section{From Poisson manifolds to symplectic microgroupoids}

3.1. Construction of $\boldsymbol{\Sigma}$. The extended symplectic "category" can be used as a target category to "symplectify" Poisson geometry via the symplectization "functor" as studied by Fernandes in [11]. The general idea is to replace an integrable Poisson manifold $(A, \Pi)$ by its source 1-connected symplectic groupoid $\Sigma(A) \rightrightarrows A$ as described in the previous section. A Poisson map $\phi:\left(B, \Pi_{B}\right) \rightarrow\left(A, \Pi_{A}\right)$ is then replaced by the Lagrangian subgroupoid $\Sigma(\phi) \subset \overline{\Sigma(A)} \times \Sigma(B)$ that integrates the graph of $\phi$ regarded as a coisotropic submanifold of $\overline{\Pi_{B}} \times \Pi_{A}$. This yields a "functor"

$$
\Sigma: \text { Poisson }^{\text {int }} \rightarrow \text { Sympl }^{\text {ext }}
$$

from the category of integrable Poisson manifolds (i.e., the Poisson manifolds that have a symplectic groupoid integrating them) to the extended symplectic "category:" the symplectization functor.

The definition of $\Sigma$ on morphisms rests on the following facts (see [5]):

- Let $G \rightrightarrows A$ be a source 1-connected symplectic groupoid over the Poisson manifold $A$ and let $L \rightrightarrows C$ be a (immersed) subgroupoid of $G \rightrightarrows A$, then $C$ is a coisotropic submanifold of $A$; conversely, there is a unique (immersed) source 1-connected Lagrangian subgroupoid that has a given coisotropic submanifold as its unit space;

\footnotetext{
${ }^{3}$ In reality, this strategy is too naive. One should first build an intermediate category that has the same objects as the microsymplectic category but whose morphisms are enhanced by half densities on the Lagrangian germs of the symplectic micromorphisms (see $[\mathbf{1}])$.
} 
- A smooth map $\phi: B \rightarrow A$ between the Poisson manifolds $A$ and $B$ is Poisson iff its graph is a coisotropic submanifold of $A \times \bar{B}$.

Therefore, we define the value of $\Sigma$ on a Poisson map $\phi: B \rightarrow A$ to be the unique Lagrangian subgroupoid $\Sigma(\phi) \rightrightarrows$ gr $\phi$ of the symplectic groupoid product $\overline{\Sigma(A)} \times \Sigma(B) \rightrightarrows A \times B$ that integrates the coisotropic submanifold gr $\phi$ of the Poisson manifold $\bar{A} \times B$.

Actually, there is a better suited "category" than $\mathbf{S y m p l}{ }^{\text {ext }}$ as target category for the symplectization "functor." Namely, consider the "category"

\section{SymplGpd ${ }^{\text {ext }}$}

whose objects are the source 1-connected symplectic groupoids. A morphism from $G \rightrightarrows A$ to $H \rightrightarrows B$ is a Lagrangian subgroupoid $L \rightrightarrows C$ of the symplectic groupoid product $\bar{G} \times H \rightrightarrows A \times B$. The composition is given by the composition of the canonical relations $L$ underlying the morphisms $L \rightrightarrows C$.

3.2. Micro-version. The symplectization functor as defined above suffers from two major problems: it is only a "functor" and it is relevant only for the class of integrable Poison manifolds. In order to define an honest symplectization functor whose domain is the category of all Poisson manifolds, we need to consider the integration of Poisson manifolds by local symplectic groupoids instead of global ones. What changes now is that every Poisson manifold can be integrated by a local symplectic groupoid $[\mathbf{7}, \mathbf{1 8}]$, or more conveniently, by a symplectic microgroupoid.

Recall that a local groupoid $G \rightrightarrows A$ is, roughly, a groupoid whose structure maps are defined only in a neighborhood of the unit space (see $[\mathbf{7 , 2 1}]$ for more details). Two local groupoids over $A$ coinciding on a (smaller) neighborhood of $A$ are equivalent for most purposes. Thus, the relevant information is really contained in the "groupoid germ" induced by the local groupoids. We are led to the following definition:

Definition 1. A (symplectic) microgroupoid $[G, A] \rightrightarrows A$ over $A$ is an equivalence class of local (symplectic) groupoids over $A$. A (Lagrangian) submicrogroupoid $[L, C] \rightrightarrows C$ of a (symplectic) microgroupoid $[G, A] \rightrightarrows A$ is a (Lagrangian) submicrofold $[L, C]$ in $[G, A]$ such that it is also a submicrogroupoids over $C$.

Now, every Poisson manifold $(A, \Pi)$ has a symplectic microgroupoid integrating it (e.g., the one constructed by Karasev in $[\mathbf{1 8}]$ ), which we will denote by

$$
[\Sigma(A), A] \rightrightarrows A,
$$

and which gives the value of the micro version of the symplectization functor

$$
\Sigma: \text { Poisson } \rightarrow \text { Sympl }_{\text {mic }}^{\text {ext }}
$$


on objects. As for the morphism component of the functor, the local Lagrangian groupoid $\Sigma(\phi) \rightrightarrows$ gr $\phi$ integrating the graph of a Poisson map $\phi: B \rightarrow A$ (as a coisotropic manifold) yields a germ of a canonical relation $[\Sigma(\phi), \operatorname{gr} \phi]$ from the symplectic microfold $[\Sigma(A), A]$ to the symplectic microfold $[\Sigma(B), B]$. Note that $[\Sigma(\phi)$, gr $\phi]$ also defines a Lagrangian submicrogroupoid over gr $\phi$, which is unique. To show that $\Sigma$ is well-defined, we still need to check that $[\Sigma(\phi), \operatorname{gr} \phi]$ is a symplectic micromorphism and that $\Sigma$ is functorial.

Proposition 2. Let $[G, A] \rightrightarrows A$ and $[H, B] \rightrightarrows B$ be two symplectic microgroupoids. Any Lagrangian submicrogroupoid $[L, \operatorname{gr} \phi] \rightrightarrows \operatorname{gr} \phi$ of the symplectic microgroupoid product, and where $\phi: B \rightarrow A$ is a smooth map, yields the symplectic micromorphism $([L], \phi):[G, A] \rightarrow[H, B]$.

Moreover, if we have another symplectic microgroupoid $[I, C] \rightrightarrows C$ and a Lagrangian submicrogroupoid $([K], \psi):[H, B] \rightarrow[I, C]$, the composition of the corresponding symplectic micromorphisms yields a Lagrangian submicrogroupoid

$$
[K \circ L, \operatorname{gr} \phi \circ \psi] \rightrightarrows \mathrm{gr} \phi \circ \psi .
$$

Proof. Let $L \rightrightarrows \operatorname{gr} \phi$ be a local groupoid representative of $[L, \operatorname{gr} \phi] \rightrightarrows \operatorname{gr} \phi$. Since $\operatorname{gr} \phi$ is the space of units of $L$, we have by definition that $L \cap(A \times$ $B)=\operatorname{gr} \phi$. We need to show that this intersection is clean ( $[\mathbf{9}$, Thm. 17]). For this, consider the local tangent groupoid $T L \rightrightarrows T \operatorname{gr} \phi$. Since $T \operatorname{gr} \phi$ is now the unit space of $T L$, we obtain for the same reason as before that $T L \cap(T A \cap T B)=T \operatorname{gr} \phi$.

The fact that $[K \circ L, \operatorname{gr} \phi \circ \psi]$ is a Lagrangian microsubgroupoid over $\operatorname{gr} \phi \circ \psi$ comes from the facts that $[L \times K$, gr $\phi \times \operatorname{gr} \psi]$ is a Lagrangian submicrogroupoid of

$$
[G \times H \times H \times I, A \times B \times B \times C] \rightrightarrows A \times B \times B \times C
$$

and that all the defining properties of a Lagrangian submicrogroupoid are preserved by the reduction with respect to $G \times \Delta_{H} \times I$ (which is directly checked by taking representatives).

The functoriality of $\Sigma$ now follows from the uniqueness of the Lagrangian submicrogroupoid in

$$
[\overline{\Sigma(A)} \times \Sigma(B), A \times B] \rightrightarrows A \times B
$$

that integrates a given Poisson map $\phi: B \rightarrow A$, and the fact that, from Proposition 2, both Lagrangian submicrogroupoids

$$
\left[\Sigma\left(\phi_{1} \circ \phi_{2}\right), \operatorname{gr} \phi_{1} \circ \phi_{2}\right] \rightrightarrows \operatorname{gr} \phi_{1} \circ \phi_{2} \leftleftarrows\left[\Sigma\left(\phi_{2}\right) \circ \Sigma\left(\phi_{1}\right), \operatorname{gr} \phi_{1} \circ \phi_{2}\right]
$$

integrate the Poisson map $\phi_{1} \circ \phi_{2}$. 
3.3. Changing the target category. Thanks to Proposition 2, we can consider the category

$$
\text { SymplGpd mic }
$$

that has the symplectic microgroupoids as its objects and whose morphisms from $[G, A] \rightrightarrows A$ to $[H, B] \rightrightarrows B$ are the symplectic micromorphisms $([L], \phi)$ from $[G, A]$ to $[H, B]$ whose underlying Lagrangian submicrofold are Lagrangian submicrogroupoids $[L, \operatorname{gr} \phi] \rightrightarrows \operatorname{gr} \phi$ of the symplectic microgroupoid product.

As in the macro case, this category can again be taken as the target category of the symplectization functor. Namely, by Proposition 2,

$$
\left(\left[\Sigma\left(\phi_{1}\right) \circ \Sigma\left(\phi_{2}\right)\right], \phi_{1} \circ \phi_{2}\right)
$$

is also a Lagrangian subgroupoid that integrates the composition of the Poisson maps $\phi_{1}$ and $\phi_{2}$. Now, in the micro case, a nice thing happens:

Theorem 3. The functor $\Sigma:$ Poisson $\rightarrow$ SymplGpd $\mathbf{d}_{\text {mic }}^{\text {ext }}$ is an equivalence of symmetric monoidal categories.

Proof. Since there is a one-to-one correspondence between the Lagrangian submicrogroupoids of the form

$$
[L, \operatorname{gr} \phi] \rightrightarrows \operatorname{gr} \phi \subset[\overline{\Sigma(A)} \times \Sigma(B), A \times B] \rightrightarrows A \times B
$$

and the Poisson maps $\phi: B \rightarrow A$, we have that $\Sigma$ is full and faithful. The essential surjectivity of $\Sigma$ on the objects follows from the fact that two symplectic microgroupoids that integrate the same Poisson manifold are related by a germ of symplectic microgroupoid isomorphism. In particular, for any symplectic microgroupoid $[G, A] \rightrightarrows A$, there is a symplectic microgroupoid isomorphism germ $[\Psi]:[G, A] \rightarrow[\Sigma(A), A]$, whose restriction to the core is the identity. Now, one checks that $\left[\operatorname{gr} \Psi, \mathrm{id}_{A}\right]$ is a Lagrangian

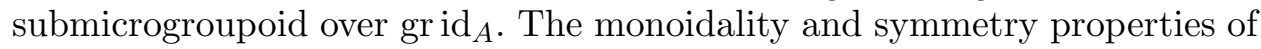
the functor come from the natural isomorphisms $\Sigma(A \times B) \simeq \Sigma(A) \times \Sigma(B)$ and $\Sigma\left(\phi_{1} \times \phi_{2}\right) \simeq \Sigma\left(\phi_{1}\right) \times \Sigma\left(\phi_{2}\right)$.

\section{From symplectic microgroupoids to monoids}

Given a symplectic groupoid $G \rightrightarrows A$, the graph of its product together with its space of units yield canonical relations gr $m: G \otimes G \rightarrow G$ and $e_{A}:\{\star\} \rightarrow G$, where $e_{A}=\{\star\} \times A$. As noted in $[\mathbf{1}, \mathbf{7}, \mathbf{3 0}]$, the triple

$$
M(G):=\left(G, \operatorname{gr} m, e_{A}\right)
$$

can be interpreted as a monoid object in the extended symplectic "category." In [30] Zakrzewski constructed a "functor"

$$
Z: \text { Poisson }^{\text {int }} \rightarrow \operatorname{Mon}\left(\operatorname{Sympl}^{\text {ext }}\right)
$$


directly from the category of integrable Poisson manifolds and complete Poisson maps to the "category" of monoids in $\mathbf{S y m p l}{ }^{\mathbf{e x t}}$ (although using a quite different language). At the level of objects, this "functor" takes an integrable Poisson manifold $(A, \Pi)$ to the monoid object $M(\Sigma(A))$ associated with its source 1-connected symplectic groupoid $\Sigma(A) \rightrightarrows A$. At the level of morphisms, Zakrzewski obtained the canonical relation corresponding to the monoid morphism directly out of the complete Poisson map by a flow construction.

In this section, we give a micro-version of $Z$, which is an honest functor and whose domain is the category of all Poisson manifolds and all Poisson maps. We obtain $Z$ as factored through the symplectization functor

$$
\text { Poisson } \left.\stackrel{\Sigma}{\longrightarrow} \text { SymplGpd } \text { mic }_{\text {ext }}^{\text {M }} \stackrel{M o n}{\longrightarrow} \text { Sympl }{ }_{\text {mic }}^{\text {ext }}\right) \text {. }
$$

We show that it yields an equivalence of symmetric monoidal categories between the category of Poisson manifolds and Poisson maps and the category of monoids and monoid morphisms in the microsymplectic category (Theorem 10).

4.1. Construction of $\boldsymbol{M}$. The association $M$ that takes a symplectic groupoid $G \rightrightarrows A$ to the corresponding monoid object $M(G)$ in the extended symplectic "category" has its corresponding version in the micro-world.

Proposition 4. The graph of the product in a symplectic microgroupoid $[G, A] \rightrightarrows A$ is a symplectic micromorphism

$$
\operatorname{gr}[m]:=\left([\operatorname{gr} m], \Delta_{A}\right):[G, A] \otimes[G, A] \rightarrow[G, A],
$$

where $\Delta_{A}: A \rightarrow A \times A$ is the diagonal map. Moreover,

$$
M([G, A]):=\left([G, A], \operatorname{gr}[m], e_{A}\right),
$$

where $e_{A}=\left(\{\star\} \times A, p r_{A}\right)$ is the unique symplectic micromorphism from the unit object $E=T^{*}\{\star\}$ to $[G, A]$, is monoid object in the microsymplectic category.

Proof. We need to check that there is a representative gr $m$ of $[\mathrm{gr} m$ ] that intersects $A^{3}$ cleanly in $\operatorname{gr} \Delta_{A}$. The fact that $(\operatorname{gr} m) \cap A^{3}=\operatorname{gr} \Delta_{A}$ comes from the facts that $m\left(a, a^{\prime}\right)=a^{\prime \prime}$ for $a, a^{\prime}, a^{\prime \prime} \in A$ iff $a=a^{\prime}=a^{\prime \prime}$. The cleanliness of the intersection comes from the repetition of this argument for the tangent local groupoid $T G \rightrightarrows T A$. The monoid object axioms follow directly from the local groupoid axioms for a representative $G \rightrightarrows A$ of the symplectic microgroupoid.

The next proposition shows that $M$ is the "object" component of a functor

$$
M: \operatorname{SymplGpd} \text { mic }_{\text {ext }} \longrightarrow \operatorname{Mon}\left(\operatorname{Sympl}_{\text {mic }}^{\text {ext }}\right) .
$$


Proposition 5. Let $[G, A] \rightrightarrows A$ and $[H, B] \rightrightarrows B$ be two symplectic microgroupoids. There is a one-to-one correspondence between the set of monoid morphisms

$$
([L], \phi): M([G, A]) \longrightarrow M([H, B])
$$

and the set of Lagrangian submicrogroupoids of the form

$$
(([L, \operatorname{gr} \phi] \rightrightarrows \operatorname{gr} \phi) \subset([\bar{G} \times H, A \times B] \rightrightarrows A \times B),
$$

where $\phi: B \rightarrow A$ is a smooth map.

Proof.

(1) Suppose that $[L, \operatorname{gr} \phi] \rightrightarrows \operatorname{gr} \phi$ is a Lagrangian submicrogroupoid as above. Let us show that the corresponding symplectic micromorphism $([L], \phi)$ is a monoid morphism. The unitality axiom (2.4) follows from the fact that there a unique symplectic micromorphism from $E$ to any given symplectic microfold; thus, $e_{B}=([L], \phi) \circ e_{A}$. As for the associativity axiom (2.3), we have to check that

$$
\left(\left[L \circ \operatorname{gr}\left[m_{G}\right], \Delta_{A} \circ \phi\right)=\left(\left[\operatorname{gr}\left[m_{H}\right] \circ(L \times L),(\phi \times \phi) \circ \Delta_{B}\right) .\right.\right.
$$

Since the core maps coincide, it is enough to show that one of the Lagrangian submicrofolds is included in the other. We do this by taking a representative $L \in[L]$ and by showing that $L\left(g_{1}\right) L\left(g_{2}\right) \subset$ $L\left(g_{1} g_{2}\right)$. Take $h_{1} h_{2} \in L\left(g_{1}\right) L\left(g_{2}\right)$, which implies that $\left(g_{1}, h_{1}\right) \in L$ and $\left(g_{2}, h_{2}\right) \in L$. Since $L$ is a subgroupoid, we also have $\left(g_{1} g_{2}, h_{1} h_{2}\right) \in L$, which, in other words, means that $h_{1} h_{2} \in L\left(g_{1} g_{2}\right)$.

(2) Suppose now that $([L], \phi)$ is a monoid morphism. Let us prove that $[L, \operatorname{gr} \phi]$ is a Lagrangian submicrogroupoid over $\operatorname{gr} \phi$. We now need to show that, for a representative $L \in[L]$, the images $\left(s_{G} \times s_{H}\right)(L)$ and $\left(t_{G} \times t_{H}\right)(L)$ coincide with $L_{0}=\operatorname{gr} \phi=L \cap(A \times B)$. For this, we repeat an argument of Zakrzewski [29, Lemma 2.4]. First of all, we immediately have

$$
\begin{aligned}
& L_{0}=\left(s_{G} \times s_{H}\right)\left(L_{0}\right) \subseteq\left(s_{G} \times s_{H}\right)(L), \\
& L_{0}=\left(t_{G} \times t_{H}\right)\left(L_{0}\right) \subseteq\left(t_{G} \times t_{H}\right)(L) .
\end{aligned}
$$

Let us check the first converse inclusion for the source maps. Take $(g, h) \in L$; we want to show that $\left(s_{G}(g), s_{H}(h)\right) \in L_{0}$. Since $\{g\}=\left(\operatorname{gr} m_{A}\right)\left(s_{G}(g), g\right)$, and $L$ is a monoid morphism, it follows that

$$
\begin{aligned}
h \in L(g) & =L\left(\left(\operatorname{gr} m_{A}\right)\left(s_{G}(g), g\right)\right), \\
& =\left(\operatorname{gr} m_{B}\right)\left(L_{0}\left(s_{G}(g)\right), L(g)\right) .
\end{aligned}
$$

This means that the source of $h$ is contained in the subset $L_{0}\left(s_{G}(g)\right)$, or, in other words, that $\left(s_{G}(g), s_{H}(h)\right) \in L_{0}$. We argue in a similar way for the target inclusion. 
It remains to show that $L \rightrightarrows$ gr $\phi$ is closed under the groupoid product: Take $\left(g_{1}, h_{1}\right) \in L$ and $\left(g_{2}, h_{2}\right) \in L$, then $h_{1} h_{2} \in L\left(g_{1}\right) L\left(g_{2}\right)=L\left(g_{1} g_{2}\right)$ since $L$ is a monoid map, which means that $\left(h_{1} h_{2}, g_{1} g_{2}\right) \in L$ and which ends the proof.

From Proposition 5, we see that the functor $M$ is a full embedding of SymplGpd mic into the category of monoids in the microsymplectic category.

4.2. Equivalence of categories. Composing now the full embedding $M$ constructed in the previous paragraph with the symplectization functor (which is an equivalence of categories)

\section{Poisson $\stackrel{\Sigma}{\longrightarrow}$ SymplGpd mic $\stackrel{\text { Mt }}{\longrightarrow} \operatorname{Mon}\left(\right.$ Sympl mic $\left._{\text {mic }}^{\text {ext }}\right)$,}

we obtain a full embedding $Z=M \circ \Sigma$, which we call the Zakrzewski functor.

At this point, we can ask if $Z$ is an equivalence of categories, or, in other words, if every monoid in the microsymplectic category arises from a symplectic microgroupoid. As the following example makes it clear, this is not true in the macro case. Namely, a symplectic groupoid has an additional structure that is not encoded in its corresponding monoid: The graph of its inverse map endows the corresponding monoid with the structure of a $\star$-monoid (see $[4,29,30]$ for a definition of a $\star$-monoid). The monoid, in the macro case, merely encodes the information relative to the product, the source and the target maps of the symplectic groupoid as well as its unit space.

Example 6. We give here a monoid object in Sympl ${ }^{\text {ext }}$ that does not come from a symplectic groupoid. Consider the operation $m\left(x_{1}, x_{2}\right)=x_{1} x_{2}$ on real numbers. We regard its cotangent lift $T^{*} m$ as a canonical relation from $\mathbb{R}^{2} \otimes \mathbb{R}^{2}$ to $\mathbb{R}^{2}$, where the symplectic structure on $\mathbb{R}^{2}$ comes from the identification $T^{*} \mathbb{R}_{x} \simeq \mathbb{R}_{x} \oplus \mathbb{R}_{y}$. Explicitly, we have

$$
T^{*} m:=\left\{\left(\left(x_{1}, y x_{2}\right),\left(x_{2}, y x_{1}\right),\left(x_{1} x_{2}, y\right)\right): x_{1}, x_{2}, y \in \mathbb{R}\right\} .
$$

One may verify directly the associativity of $T^{*} m$, which follows from that of $m$. There is a unit, which is given by the Lagrangian submanifold $E=$ $\{(1, y): y \in \mathbb{R}\}$. We still have a source and a target, which coincide here

$$
s=t=\pi: \mathbb{R}^{2} \rightarrow E ;(x, y) \mapsto(1, x y) .
$$

The restriction of $T^{*} m$ to the fibers of $\pi$ over points $(1, \alpha)$ with $\alpha \neq 0$ can be seen as the graph of a product

$$
\left(x_{1}, \frac{\alpha}{x_{1}}\right) \bullet\left(x_{2}, \frac{\alpha}{x_{2}}\right)=\left(x_{1} x_{2}, \frac{\alpha}{x_{1} x_{2}}\right) .
$$




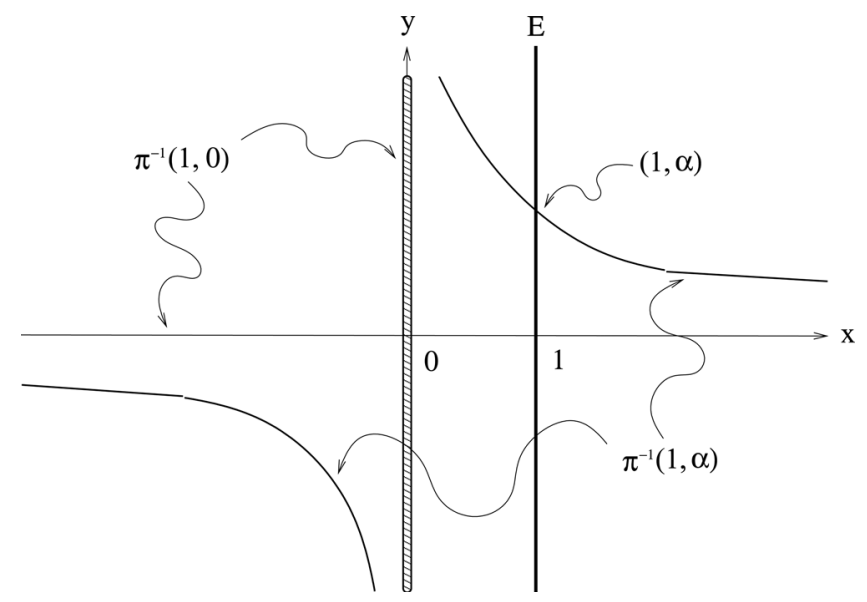

Figure 1. The source (and target) fibre $\pi^{-1}(1,0)$ is singular.

Actually, this product endows the fiber $\pi^{-1}(1, \alpha)$, which is the hyperbola $H_{\alpha}=\{(x, y): x y=\alpha\}$, as represented in Figure 1, with a group structure. The unit is the element $(1, \alpha)$, and the inverse is

$$
\left(x, \frac{\alpha}{x},\right)^{-1}=\left(\frac{1}{x}, \alpha x\right) .
$$

Although we are very close to having a symplectic groupoid, there is a "singular" fiber; $\pi^{-1}((1,0))$ is the union of the two intersecting lines $l_{x}=\{(x, 0): x \in \mathbb{R}\}$ and $l_{y}=\{(0, y): y \in \mathbb{R}\}$. The product is defined on $l_{x} \backslash\{(0,0)\}$ as the usual real multiplication, but it is not defined at all on $l_{y} \backslash\{(0,0)\}$. Moreover, the product is multi-valued at $(0,0)$; namely,

$$
(0,0) \bullet(0,0)=\{(0, y): y \in \mathbb{R}\} .
$$

Observe that the problematic points (i.e., the whole line $l_{y}$ ) coincide with the locus of points where the inverse is not defined.

The situation in the micro-world is different. The following example illustrates the fact that, even though a monoid object in the extended symplectic category may not arise from a symplectic groupoid, its restriction to a (micro) neighborhood of its unit space yields a monoid object in the microsymplectic category, which itself comes from a symplectic microgroupoid. This is essentially due to the fact that all the structure maps (including the inverse map) of a local symplectic groupoid can be recovered from the source map alone $[\mathbf{7 , 1 8}]$.

Example 7. Let us look at Example 6 again. The monoid $\left(\mathbb{R}^{2}, T^{*} m, E\right)$ in $\mathbf{S y m p l}^{\text {ext }}$ fails to come from a symplectic groupoid because the natural 
inverse map

$$
i(x, y)=\left(\frac{1}{x}, y x^{2}\right)
$$

can not be extended to the line $l_{y}$ of the "singular" fiber $\pi^{-1}(1,0)=l_{x} \cup l_{y}$. This prevents this monoid from having a $\star$-monoid structure. However, if we look at the restriction of $T^{*} m$ to a neighborhood

$$
U_{\epsilon}:=\{(1+\eta, y): \eta \in(-\epsilon, \epsilon), y \in \mathbb{R}\}, \quad 0<\epsilon<1,
$$

we obtain a local symplectic groupoid over $E$ and a corresponding symplectic micromorphism

$$
\left(\left[T^{*} m\right], \Delta_{E}\right):\left[\mathbb{R}^{2}, E\right] \otimes\left[\mathbb{R}^{2}, E\right] \rightarrow\left[\mathbb{R}^{2}, E\right],
$$

turning $\left[\mathbb{R}^{2}, E\right]$ into a monoid object in the microsymplectic category.

First of all, we will use the coordinates $q:=y$ on $E$ and $p:=x-1$ on the vertical fiber so that a point $\left(x, \frac{\alpha}{x}\right)$ in the $x y$-coordinates reads $\left(x-1, \frac{\alpha}{x}\right)$ in the $p q$-coordinates. Now the restriction of $T^{*} m$ to $U_{\epsilon}$ becomes

$$
\left\{\left(\left(p_{1}, q\left(p_{2}+1\right)\right),\left(p_{2}, q\left(p_{1}+1\right)\right),\left(p_{1}+p_{2}+p_{1} p_{2}, q\right): 0<p_{1}, p_{2}<1, q \in \mathbb{R}\right\} .\right.
$$

In these coordinates, the source and target, which coincide, of the local symplectic groupoid $U_{\epsilon} \rightrightarrows E$ are given by the map $\pi(p, q)=q(p+1)$. Now, for each $\alpha \in \mathbb{R}$, the fiber

$$
\pi^{-1}(\alpha)=\left\{\left(p, \frac{\alpha}{p+1}\right): p \in(-\epsilon, \epsilon)\right\}
$$

is an honest submanifold, and the groupoid product and the inverse map, restricted to this fiber, are given by

$$
\begin{aligned}
\left(p_{1}, \frac{\alpha}{p_{1}+1}\right) \bullet & \left(p_{2}, \frac{\alpha}{p_{2}+1}\right)=\left(p_{1}+p_{2}+p_{1} p_{2}, \frac{\alpha}{p_{1}+p_{2}+p_{1} p_{2}+1}\right), \\
\left(p, \frac{\alpha}{p+1}\right)^{-1} & =\left(\frac{-p}{p+1}, \frac{\alpha}{\left(\frac{-p}{p+1}\right)+1}\right) .
\end{aligned}
$$

Figure 2 shows the local groupoid fibration generated by the family of hyperbolae $H_{\alpha}$, for $\alpha \neq 0$, together with the line $l_{x}$ in a neighborhood of the unit space $E$ in Example 6.

Actually, in the micro case, we have the following proposition:

Proposition 8. Every monoid in the microsymplectic category comes from a symplectic microgroupoid.

Proof. To begin with, we show that $\mu=\operatorname{gr}[m]$, where $[m]$ is the germ of a map $m: \mathcal{C} \rightarrow G$, where $\mathcal{C}$ is a representative of a coisotropic submicrofold 


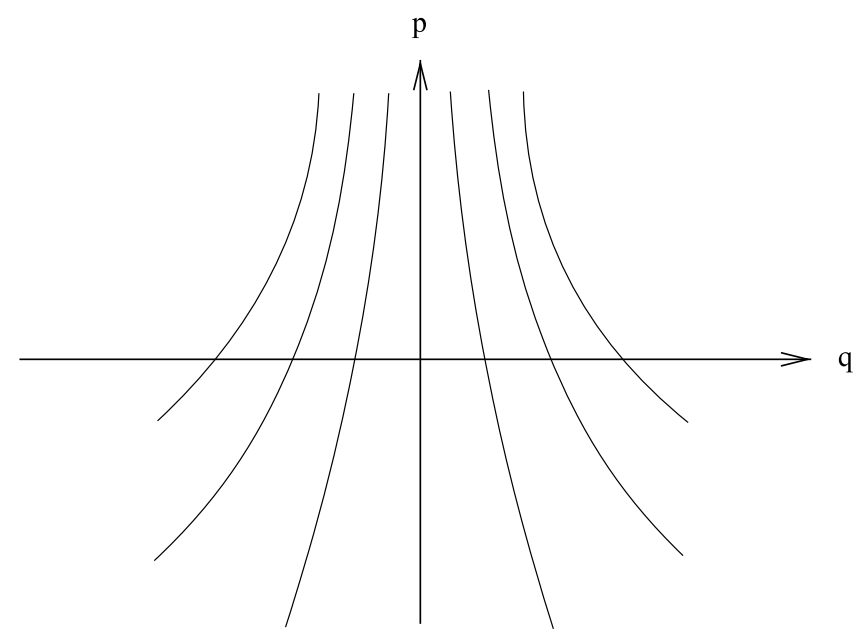

Figure 2. The monoid of Example 6 is a local symplectic groupoid if restricted to a neighborhood of the units.

$\left[\mathcal{C}, \operatorname{grid}_{A}\right] \subset[G \times G, A \times A]$. To see this, we will use the result $[\mathbf{9}$, Theorem 27], which, applied to the symplectic micromorphism

$$
\mu=([V], \phi):\left[G^{2}, A^{2}\right] \rightarrow[G, A],
$$

states the following: For any collection $\mathcal{F} A:=\left\{\left[\mathcal{F}_{x} A,\{x\}\right]\right\}_{x \in A}$ of Lagrangian submanifold germs in $[G, A]$ transverse to $A$, there is a corresponding collection

$$
\mathcal{F} A^{2}:=\left\{\left[\mathcal{F}_{x} A^{2},\{\phi(x)\}\right]\right\}_{x \in A}
$$

of Lagrangian submanifold germs in $\left[G^{2}, A^{2}\right]$, each of which is transverse to the core $A^{2}$, together with a collection of map germs

$$
m_{x}: \mathcal{F}_{x} A^{2} \rightarrow \mathcal{F}_{x} A, \quad x \in A,
$$

such that, for suitable representatives,

$$
\begin{aligned}
\operatorname{gr} m_{x} & =V \cap\left(\mathcal{F}_{x} A^{2} \times \mathcal{F}_{x} A\right), \\
V & =\bigcup_{x \in A} \operatorname{gr} m_{x} .
\end{aligned}
$$

The key point now is to note that the unitality axiom (2.2) forces the core map of $\mu$ to be the diagonal map $\Delta_{A}: A \rightarrow A \times A$, which is an embedding. This implies that the Lagrangian germs in the collection $\mathcal{F} A^{2}$ are all disjoint. Therefore, their union

$$
\mathcal{C}:=\bigcup_{x \in A} \mathcal{F}_{x} A^{2}
$$

is a germ of a coisotropic submanifold around $\{(x, x): x \in A\}$, on which the collection $\left\{m_{x}\right\}_{x \in A}$ defines a map $m: \mathcal{C} \rightarrow A$ such that $V=$ gr $m$. This is 
the (local) symplectic groupoid product, and $\mathcal{C}$ is the space of composable pairs.

Now, let us extract the source, target and inverse maps while verifying the local groupoid axioms. The associativity axiom (2.1) in term of $m$ yields directly that

$$
\left(g_{1}, g_{2}\right) \in \mathcal{C},\left(m\left(g_{1}, g_{2}\right), g_{3}\right) \in \mathcal{C} \Rightarrow\left(g_{2}, g_{3}\right) \in \mathcal{C},\left(g_{1}, m\left(g_{2}, g_{3}\right)\right) \in \mathcal{C} .
$$

Moreover in this case we have that

$$
m\left(m\left(g_{1}, g_{2}\right), g_{3}\right)=m\left(g_{1}, m\left(g_{2}, g_{3}\right)\right) .
$$

Since $\operatorname{gr}[m]$ is a symplectic micromorphism with core map $\Delta: A \rightarrow A \times A$, we have that gr $m \cap(A \times A \times G)=\operatorname{gr} \Delta$. Hence, for $x_{1}, x_{2} \in A$, we have that $\left(x_{1}, x_{2}\right) \in \mathcal{C}$ iff $x_{1}=x_{2}=x$ and then $m(x, x)=x$.

We can extract the source and target maps from the fact that the composition of symplectic micromorphisms is always monic (see [9]) when applied to the compositions involved in the unitality axiom (2.2). Namely, consider the reduction

$$
\pi: G \times \Delta_{G \times G} \times G \rightarrow G \times G
$$

associated with the composition

$$
G \simeq\{*\} \times G \stackrel{e_{A} \times \mathrm{id}}{\longrightarrow} G \times G \stackrel{\text { grm }}{\longrightarrow} G .
$$

The monicity of the composition can be expressed by saying that the restriction $\pi^{\prime}$ of $\pi$ to

$$
K:=\left(A \times \Delta_{G} \times \operatorname{gr} m\right) \cap\left(G \times \Delta_{G \times G} \times G\right)
$$

is a diffeomorphism on its image gr $m \circ\left(e_{A} \otimes \mathrm{id}\right)$. Now, using the identification $K \simeq(A \times G) \cap \mathcal{C}$, the restriction $\pi^{\prime}$ reads

$$
\pi^{\prime}:(A \times G) \cap \mathcal{C} \rightarrow G \times G:(a, g) \rightarrow(g, m(a, g))=(g, g) .
$$

Taking the inverse of $\pi^{\prime}$ on its image (which is the diagonal in $G \times G$ by the unitality axiom), we obtain a smooth surjective map $s: G \rightarrow A$ for which $s(g)$ is the unique element in $A$ such that

$$
(s(g), g) \in \mathcal{C} \quad \text { and } \quad m(s(g), g)=g \quad \text { for all } \quad g \in G .
$$

In other words, we have obtained the source map of the local symplectic groupoid. The same line of argument for the other composition gr $m \circ\left(\right.$ id $\left.\otimes e_{A}\right)$ delivers the target map $t: G \rightarrow A$. Let us check now that these maps behave the way they should with respect to the product $m$; for this, we use here essentially the same arguments as in Zakrzewski [29, Lemma 2.3]. Let $\left(g_{1}, g_{2}\right) \in \mathcal{C}$, then

$$
\begin{aligned}
& m\left(g_{1}, g_{2}\right)=m\left(m\left(s\left(g_{1}\right), g_{1}\right), g_{2}\right)=m\left(s\left(g_{1}\right), m\left(g_{1}, g_{2}\right)\right), \\
& m\left(g_{1}, g_{2}\right)=m\left(g_{1}, m\left(g_{2}, t\left(g_{2}\right)\right)\right)=m\left(m\left(g_{1}, g_{2}\right), t\left(g_{2}\right)\right),
\end{aligned}
$$


which implies, by uniqueness of the source and target map, that

$$
s\left(m\left(g_{1}, g_{2}\right)\right)=s\left(g_{1}\right) \text { and } t\left(m\left(g_{1}, g_{2}\right)\right)=t\left(g_{2}\right) .
$$

Since we also have, for $\left(g_{1}, g_{2}\right) \in \mathcal{C}$, that

$$
m\left(g_{1}, g_{2}\right)=m\left(m\left(g_{1}, t\left(g_{1}\right)\right), g_{2}\right)=m\left(g_{1}, m\left(t\left(g_{1}\right), g_{2}\right)\right),
$$

which implies that $\left(t\left(g_{1}\right), g_{2}\right) \in \mathcal{C}$, we obtain that

$$
\forall\left(g_{1}, g_{2}\right) \in \mathcal{C}, \quad t\left(g_{1}\right)=s\left(g_{2}\right)
$$

because $s\left(g_{2}\right)$ is the unique element $x \in A$ such that $\left(x, g_{2}\right)$ is composable.

To extract the inverse map, we consider the restriction $m_{x}$ of the product $m$ to the fiber $\mathcal{F}_{x} A^{2}$ and the implicit equation $m_{x}\left(g_{1}, g_{2}\right)=x$. Since $m_{x}(x, x)=x$ and $\partial_{g_{1}} m_{x}(x, x)=\partial_{g_{2}} m_{x}(x, x)=\mathrm{id}$, we can apply the implicit function theorem in both arguments, obtaining two families of smooth functions, $\left\{i_{x}\right\}_{x \in A}$ and $\left\{j_{x}\right\}_{x \in A}$, such that

$$
m_{t(g)}\left(i_{t(g)}(g), g\right)=t(g) \text { and } m_{s(g)}\left(g, j_{s(g)}(g)\right)=s(g) .
$$

The collection $\left\{\mathcal{F}_{x} A^{2}\right\}_{x \in A}$ of Lagrangian submanifolds is a partition of $\mathcal{C}$, and so we can glue the two collections $\left\{i_{x}\right\}_{x \in A}$ and $\left\{j_{x}\right\}_{x \in A}$ into smooth functions $i, j: G \rightarrow G$ such that

$$
m(i(g), g)=t(g) \text { and } m(g, j(g))=s(g) .
$$

Since, by construction, $(i(g), g),(g, j(g)) \in \mathcal{C}$, we also have that

$$
t(i(g))=s(g) \text { and } t(g)=s(j(g)) .
$$

Let us show that $i$ and $j$ coincide. Namely, $(m(i(g), g), j(g)) \in \mathcal{C}$ because $m(i(g), g)=t(g)=s(j(g))$ and thus

$$
j(g)=m(m(i(g), g), j(g))=m(i(g), m(g, j(g))=i(g) .
$$

As explained in Section 2.3, the category of monoids in the microsymplectic category inherits the structure of a symmetric monoidal category.

Theorem 9. The functor $M: \mathbf{S y m p l G p d}$ mic $\rightarrow \mathbf{M o n}\left(\mathbf{S y m p l}_{\text {mic }}^{\text {ext }}\right)$ is an equivalence of symmetric monoidal categories.

Proof. Proposition 8 tells us that $M$ is an isomorphism on the objects and Proposition 5 tells us that $M$ is full and faithful. Hence, we have an isomorphism of categories.

Given two symplectic microgroupoids $[G, A] \rightrightarrows A$ and $[H, B] \rightrightarrows B$ the groupoid operation in their tensor product is given by

$$
\left[m_{G \times H}\right]:=\left(\left[m_{G}\right] \times\left[m_{H}\right]\right) \circ\left(\mathrm{id}_{G} \times \epsilon_{G, H} \times \mathrm{id}_{H}\right) .
$$

Therefore, the image by $M$ of the symplectic microgroupoid product is the monoid

$$
\left([G \times H, A \times B], \mu, e_{A \times B}\right),
$$


where

$$
\mu:=\left(\operatorname{gr}\left[m_{G}\right] \otimes \operatorname{gr}\left[m_{H}\right]\right) \circ\left(\operatorname{id}_{[G, H]} \otimes \operatorname{gr} \epsilon_{G, H} \otimes \operatorname{id}_{H}\right) .
$$

From Section 2.3, we see that this monoid coincides with the tensor product of the monoids; i.e.,

$$
M([G \times H, A \times B)=M([G, A]) \otimes M([H, B]) .
$$

A similar argument shows that we also have this property for the morphisms; i.e.,

$$
M([V \otimes W], \phi \times \psi)=M([V], \phi) \otimes M([W], \psi) .
$$

Moreover, the unit symplectic microgroupoid $[\{0\} \times\{\star\},\{\star\}] \rightrightarrows\{\star\}$ is sent on the unit monoid by $M$. Thus, we have an equivalence of monoidal categories. Checking that $M$ also respects the symmetries is straightforward.

Now, composing the following equivalences:

$$
\text { Poisson } \stackrel{\Sigma}{\longrightarrow} \text { SymplGpd } \text { mic }_{\text {ic }}^{\text {ext }} \stackrel{M}{\longrightarrow} \text { Mon }\left(\operatorname{Sympl}_{\text {mic }}^{\text {ext }}\right),
$$

we obtain the main theorem of this paper:

Theorem 10. The symmetric monoidal category of Poisson manifolds and Poisson maps is equivalent to the symmetric monoidal category of monoids in the microsymplectic category.

\section{References}

[1] S. Bates and A. Weinstein, Lectures on the geometry of quantization, Berkeley Mathematics Lecture Notes, Amer. Math. Soc. 8 (1997).

[2] P. Bieliavsky, Strict quantization of solvable symmetric spaces, J. Symplectic Geom. 1 (2002), 269-320.

[3] P. Bieliavsky, S. Detournay and P. Spindel, The deformation quantizations of the hyperbolic plane, Comm. Math. Phys. 289 (2009), 529-559.

[4] S. Canez, Double Groupoids, Orbifolds, and the Symplectic Category, PhD thesis, University of California Berkeley (2011), http://arxiv.org/abs/1105.2592v1.

[5] A.S. Cattaneo, On the integration of Poisson manifolds, Lie algebroids, and coisotropic submanifolds, Lett. Math. Phys. 67 (2004), 33-48.

[6] A.S. Cattaneo and G. Felder, Poisson sigma models and symplectic groupoids, Quantization of singular symplectic quotients, Progr. Math. 198 (2001), 61-93.

[7] A. Coste, P. Dazord and A. Weinstein, Groupoides symplectiques, Publications du Departement de Mathematiques, Nouvelle Serie A, Vol. 2, Univ. Claude-Bernard, Lyon, (1987). http://math.berkeley.edu/ alanw/cdw.pdf.

[8] A.S. Cattaneo, B. Dherin and A. Weinstein, Symplectic microgeometry I: micromorphisms, J. Symplectic Geom. 8 (2010), 205-223.

[9] A.S. Cattaneo, B. Dherin and A. Weinstein, Symplectic microgeometry II: generating functions, Proceedings of Poisson 2010, Bull. Brazilian Math. Soc. 4 (2011), 507-536.

[10] M. Crainic and R.L. Fernandes, Integrability of Poisson brackets, J. Differ. Geom. 66 (2004), 71-137. 
[11] R.L. Fernandes, The symplectization functor, XV Int. Workshop on Geometry and Physics, Publ. R. Soc. Mat. Esp. 11 (2007), 67-82.

[12] J. Gracia-Bondia and J.C. Várilly, From geometric quantization to Moyal quantization, J. Math. Phys. 36 (1995), 2691-2701.

[13] E. Hawkins, A groupoid approach to quantization, J. Symplectic Geom. 6 (2008), 61-125.

[14] A.V. Karabegov, On the dequantization of Fedosov's deformation quantization, Lett. Math. Phys. 65 (2003), 133-146.

[15] A.V. Karabegov, On the inverse mapping of the formal symplectic groupoid of a deformation quantization, Lett. Math. Phys. 70 (2004), 43-56.

[16] A.V. Karabegov, Formal symplectic groupoid of a deformation quantization, Comm. Math. Phys. 258 (2005), 223-256.

[17] A.V. Karabegov, Fedosov's formal symplectic groupoids and contravariant connections, J. Geom. Phys. 56 (2006), 1985-2009.

[18] M.V. Karasev, Analogues of the objects of Lie group theory for nonlinear Poisson brackets, Math. USSR Izvestiya 28 (1987), 497-527.

[19] M.V. Karasev and V.P. Maslov, Nonlinear Poisson brackets. Geometry and quantization, Translations of Mathematical Monographs 119, AMS, 1993.

[20] N.P. Landsman, Quantization as a functor, Quantization, Poisson brackets and beyond (Manchester, 2001), Contemp. Math. 315 (2002), 9-24.

[21] K. Mackenzie, Lie Groupoids and Lie Algebroids in Differential Geometry, London Mathematical Society Lecture Note Series 124, Cambridge University Press, 1987.

[22] S. Mac Lane, Categories for the Working Mathematician, 2nd edn, Graduate Texts in Mathematics 5, Springer-Verlag, 1998.

[23] P. de, M. Rios and G.M. Tuynman, Weyl quantization from geometric quantization, AIP Conf. Proc. (2008), 26-38.

[24] H. Tseng and C. Zhu, Integrating Poisson manifolds via stacks, Trav. Math. 16 (2005), 285-297, Univ. Luxemb.

[25] A. Weinstein, Noncommutative geometry and geometric quantization, Symplectic geometry and mathematical physics (Aix-en-Provence, 1990), Progr. Math., 99 (1991), 446-461.

[26] A. Weinstein, Traces and triangles in symmetric symplectic spaces, Contemporary Mathematics 179 (1994).

[27] A. Weinstein, Tangential deformation quantization and polarized symplectic groupoids, Deformation theory and symplectic geometry (Ascona, 1996), Math. Phys. Stud. 20 (1997), 301-314

[28] C. Woodward and K. Wehrheim, Functoriality for Lagrangian correspondences in Floer theory, Quantum Topol. 1 (2010), 129-170.

[29] S. Zakrzewski, Quantum and classical pseudogroups. Part I. Union pseudogroups and their quantization, Comm. Math. Phys. 134 (1990), 347-370.

[30] S. Zakrzewski, Quantum and classical pseudogroups. Part II. Differential and Symplectic Pseudogroups, Comm. Math. Phys. 134 (1990), 371-395. 


\author{
INSTITUT FÜR MATHEMATIK \\ UNIVERSITÄT ZÜRICH-IRCHEL \\ WinterTHURERSTRASSE 190, CH-8057 ZÜRICH \\ SWITZERLAND \\ E-mail address: alberto.cattaneo@math.uzh.ch \\ Department of Mathematics \\ University of CALIFORNIA \\ BERKELEY \\ CA 94720-3840 \\ E-mail address: dherin@math.berkeley.edu \\ Department of Mathematics \\ UNIVERSITY OF CALIFORNIA \\ BERKElEy, CA 94720-3840 \\ E-mail address: alanw@math.berkeley.edu
}

Received 10/23/2011, accepted 06/18/2012

We thank Domenico Fiorenza for useful suggestions. A.S.C. acknowledges partial support from SNF Grant 20-131813. B.D. acknowledges partial support from SNF Grant PA002-113136, NWO Grant 613.000.602 and FAPESP grant 2010/15069-8, as well as the hospitality of the ICMC of the University of São Paulo, in São Carlos. B.D. thanks Ittay Weiss for inspiring discussions about monoids in Rel. In addition, B.D. thanks Ieke Moerdijk and Marius Crainic for providing a highly stimulating research environment at Utrecht University, where part of this paper was done. A.W. acknowledges partial support from NSF grant DMS-0707137 and the hospitality of the Institut Mathématique de Jussieu. 
